\title{
THE FAT AND WATER CONTENT OF THE LEFT AND RIGHT LIVER BEFORE AND AFTER BIRTH
}

\author{
BY \\ JOHN L. EMERY and ETHEL FINCH \\ From the Department of Pathology, Children's Hospital, Sheffield
}

(RECEIVED FOR PUBLICATION DECEMBER 14, 1953)

Asymmetrical foamy-celled change in the right lobe of the liver in newborn infants was noted by Gruenwald (1949), and we have described (Emery, 1952) a rapid degeneration of the left physiological lobe of the liver following birth. These asymmetrical changes in the liver are associated with the different blood supply to the right and left physiological lobes in utero and are probably of great importance in metabolic changes in the newborn and in the study of later intra-uterine diseases. It is also obvious that the liver in the foetus and in the newborn should not be studied as a single organ but as two distinct but united organs, that is, a right and left liver.

In order to investigate this problem further we have examined the fat and water content, both histologically and chemically, of the livers of children dying prematurely, at full term during the neonatal period and in some older children. Great differences between the contents of the right and left lobes were found in many cases, but before such findings could be assessed it was necessary to study the normal variation.

Very little information is to be found in the literature on the amount of fat occurring normally in the human embryo in the neonatal period or concerning the lobe differences. Also examinations that have been reported have apparently not considered the pathological fatty changes now known to occur in the liver in utero and thus did not distinguish between normal and abnormal livers (Widdowson and Spray, 1951).

Dorkin and Weinberg (1949) studied the histologically demonstrable fat in the livers of stillborn and newborn infants. They endeavoured to correlate fat content with maturity and concluded that the storage of fat reaches its maximum during the last month of gestation. Needham (1931) had also found an increase of fat in the human embryo as a whole from five to nine months but did not indicate if liver fat as such increased over this period.

Rourke and Stewart (1942) examined the liver with regard to its uniformity with respect to the concentration of fat, water and vitamin $A$ in different parts of the same liver and concluded that on the whole fats and water are uniformly distributed. Billing, Conlon, Hein and Schiff (1953) estimated the liver lipids in man in different parts of the liver by means of specimens taken by needle biopsy. They also found relative uniformity throughout the liver and that the histology and chemical estimation of fat content paralleled each other. Most of these investigations, however, were done on livers from adults.

This communication records an attempt to establish the normal fat and water contents of the left and right livers in later foetal and early neonatal life.

\section{Materials and Methods}

For about a year the fat and water content of the left and right liver has been estimated on all (134) stillborn and neonatal infants coming to necropsy. The livers were divided into three parts by two cuts, one along the line of the anterior ligament and ductus venosus, that is, cutting off the anatomical left lobe. The second cut was taken from the tip of the gall bladder to the inferior vena cava through the base of the gall bladder to the hilum. This cut divides the liver into the physiological left and right lobes. The central part of the liver constituting the median part of the left physiological lobe was discarded after weighing. Blocks of distinctive shape were taken for histology from the left and right lobes, the remainder of which were then separately cut into thin slices by a very sharp knife and blotted to remove as much blood as possible.

For chemical analysis the whole of the sliced lobes were weighed and dried on a hot plate at $95^{\circ}-100^{\circ} \mathrm{C}$. until a constant weight was obtained. From these values the water content was calculated. After grinding the tissue to a powder, duplicate samples were weighed, 
boiled for 30 minutes in $30 \%$ hydrochloric acid, the fat extracted with ether, the ether evaporated, and the residual fat weighed after cooling in a vacuum desiccator. The fat content was then expressed as a percentage of the dry weight.

Sections from both lobes were carefully examined and livers showing any cellular abnormality in either lobe were excluded. Frozen sections were stained for fat in many instances and any liver showing fatty droplets was excluded. However, no liver was seen in paraffin sections to contain fat that did not show other changes microscopically which would in any case have caused its exclusion from the normal series. Cases showing known intra-uterine disease, such as $\mathrm{Rh}$ incompatibility and diseases of the placenta, were also excluded even when no abnormality was seen in the liver. This necessitated excluding about two-thirds of the cases examined.

The maturity of the infants was estimated on a combination of the duration of pregnancy and the crownrump, crown-heel length of the infants using the range given by Streeter (1920) and Scammon and Calkins (1923).

\section{Results}

In order to assess any significant difference between the right and left lobes it was necessary to analyse the differences between duplicates of each lobe.

It was found that the range of difference from duplicate fat estimations on 96 specimens was 0-1.6 g. per $100 \mathrm{~g}$. dry liver, with a mean difference of $0.43 \mathrm{~g}$. (standard deviation 0.47 ). From these figures any difference greater than $1.88 \mathrm{~g}$. \% was regarded as significant.

Similarly the duplicate analyses of water content were statistically analysed with the following results. There was a range of difference from 0 to $1.7 \%$ with a mean difference of 0.3 (standard deviation
$0 \cdot 3)$. Therefore any figures varying by more than $1 \cdot 2 \%$ may be regarded as significantly different.

Water Content of Liver before Birth. No significant difference was found in the water content of the livers from foetuses of six, seven, eight and nine months' gestation or in water content of the left and right lobes at any age (Table 1). In livers of 10 or more months' gestation there appeared to be a small diminution in water content affecting the liver symmetrically.

The variation in water content within an age group was wider than that between different age groups. All livers showing a water content of $80 \%$ or more were from 'immature' infants. The difference in water content of different livers in the same age group was much greater than any difference between the different lobes of the same liver.

Water Content of Livers after Birth. Since infants of different maturity appeared to have differing liver water contents it would seem ideally desirable to study post-natal changes in different maturity groups. The data, however, for this were much too small, hence the whole series of live births is considered together in Table 2 and Fig. 1.

A survey of the figures showed no significant changes in water content with age, as wider variations occurred in each group than between different age groups. On the other hand, water contents of $79 \%$ and over were only found in infants under the age of 12 hours. But these levels are themselves explicable on the immaturity of some of the individuals constituting this group. There would seem to be no gross diminution in water content after birth.

TABLE 1

WATER CONTENT OF RIGHT AND LEFT LOBES OF THE LIVER OF INFANTS DYING BEFORE THE AGE OF 24 HOURS RELATED TO MATURITY

\begin{tabular}{|c|c|c|c|c|c|c|c|c|c|c|c|}
\hline $\begin{array}{c}\text { Maturity } \\
\text { (months) }\end{array}$ & & & Percentage & Water & Content of & Left and & Right Lo & & & Range & Average \\
\hline 6 & $\begin{array}{ll}\text { R. } & 77 \cdot 6 \\
\text { L. } & 77 \cdot 8\end{array}$ & & & & & & & & & - & $\begin{array}{l}77 \cdot 6 \\
77 \cdot 8\end{array}$ \\
\hline 7 & $\begin{array}{ll}\text { R. } & 75 \cdot 9 \\
\text { L. } & 75 \cdot 0\end{array}$ & $\begin{array}{l}85 \cdot 1 \\
86 \cdot 0\end{array}$ & $\begin{array}{l}73 \cdot 6 \\
74 \cdot 8\end{array}$ & & & & & & & $\begin{array}{l}73 \cdot 6-85 \cdot 1 \\
74 \cdot 8-86-0\end{array}$ & $\begin{array}{l}78 \cdot 2 \\
78 \cdot 6\end{array}$ \\
\hline 8 & $\begin{array}{ll}\text { R. } & 78 \cdot 7 \\
\text { L. } & 78 \cdot 4\end{array}$ & $\begin{array}{l}75 \cdot 6 \\
78 \cdot 3\end{array}$ & $\begin{array}{l}76 \cdot 1 \\
77 \cdot 3\end{array}$ & $\begin{array}{l}75 \cdot 2 \\
75 \cdot 9\end{array}$ & $\begin{array}{l}79 \cdot 1 \\
79 \cdot 1\end{array}$ & $\begin{array}{l}79 \cdot 2 \\
78 \cdot 8\end{array}$ & $\begin{array}{l}79 \cdot 7 \\
80 \cdot 4\end{array}$ & $\begin{array}{l}80 \cdot 1 \\
80 \cdot 2\end{array}$ & $\begin{array}{l}77 \cdot 5 \\
77 \cdot 7\end{array}$ & $\begin{array}{l}75 \cdot 2-80 \cdot 1 \\
75 \cdot 9-80 \cdot 4\end{array}$ & $\begin{array}{l}77 \cdot 9 \\
78 \cdot 5\end{array}$ \\
\hline 9 & $\begin{array}{ll}\text { R. } & 76 \cdot 8 \\
\text { L. } & 76 \cdot 8\end{array}$ & $\begin{array}{l}80 \cdot 0 \\
79 \cdot 0\end{array}$ & $\begin{array}{l}76 \cdot 4 \\
77 \cdot 0\end{array}$ & & & & & & & $\begin{array}{l}76 \cdot 4-80 \cdot 0 \\
76 \cdot 8-79 \cdot 0\end{array}$ & $\begin{array}{l}77 \cdot 7 \\
77 \cdot 6\end{array}$ \\
\hline \multirow[t]{2}{*}{10} & $\begin{array}{ll}\text { R. } & 76 \cdot 5 \\
\text { L. } & 74 \cdot 7\end{array}$ & $\begin{array}{l}74 \cdot 0 \\
75 \cdot 3\end{array}$ & $\begin{array}{l}74 \cdot 5 \\
75 \cdot 5\end{array}$ & $\begin{array}{l}71 \cdot 8 \\
72 \cdot 7\end{array}$ & $\begin{array}{l}75 \cdot 0 \\
73 \cdot 8\end{array}$ & $\begin{array}{l}76 \cdot 0 \\
76 \cdot 6\end{array}$ & \multirow[t]{2}{*}{$\begin{array}{l}76 \cdot 1 \\
76 \cdot 5\end{array}$} & \multirow[t]{2}{*}{$\begin{array}{l}75 \cdot 2 \\
74 \cdot 2\end{array}$} & \multirow[t]{2}{*}{$\begin{array}{l}78 \cdot 2 \\
76 \cdot 8\end{array}$} & \multirow{2}{*}{$\begin{array}{l}71 \cdot 8-78 \cdot 7 \\
72 \cdot 2-78 \cdot 0\end{array}$} & \multirow{2}{*}{$\begin{array}{l}75 \cdot 5 \\
75 \cdot 5\end{array}$} \\
\hline & $\begin{array}{ll}\text { R. } & 72.6 \\
\text { L. } & 73.6\end{array}$ & $\begin{array}{l}78 \cdot 7 \\
77 \cdot 7\end{array}$ & $\begin{array}{l}74 \cdot 3 \\
75 \cdot 3\end{array}$ & $\begin{array}{l}76 \cdot 9 \\
77 \cdot 2\end{array}$ & $\begin{array}{l}75 \cdot 2 \\
74 \cdot 9\end{array}$ & $\begin{array}{l}78 \cdot 1 \\
78 \cdot 0\end{array}$ & & & & & \\
\hline 11 & $\begin{array}{ll}\text { R. } & 74 \cdot 0 \\
\text { L. } & 73 \cdot 8\end{array}$ & $\begin{array}{l}74 \cdot 0 \\
73 \cdot 5\end{array}$ & & & & & & & & $\begin{array}{c}74 \cdot 0 \\
73 \cdot 5-73 \cdot 8\end{array}$ & $\begin{array}{l}74 \cdot 0 \\
73 \cdot 7\end{array}$ \\
\hline
\end{tabular}


TABLE 2

WATER CONTENT OF LIVER RELATED TO THE IMMEDIATE POST-NATAL AGE OF THE CHILD AT DEATH

\begin{tabular}{|c|c|c|c|c|c|c|c|c|c|c|}
\hline \multicolumn{2}{|l|}{ Age } & \multicolumn{7}{|c|}{ Percentage Water Content } & \multirow{2}{*}{$\begin{array}{c}\text { Range } \\
74 \cdot 0-80 \cdot 1 \\
73 \cdot 2-80 \cdot 2\end{array}$} & \multirow{2}{*}{$\begin{array}{c}\text { Average } \\
76 \cdot 5 \\
76 \cdot 4\end{array}$} \\
\hline Birth-5 hours & .. & $\begin{array}{l}\mathbf{R} \text {. } \\
\mathbf{L} \text {. }\end{array}$ & $\begin{array}{l}74 \cdot 0 \\
73 \cdot 2\end{array}$ & $\begin{array}{l}74 \cdot 0 \\
73 \cdot 8\end{array}$ & $\begin{array}{l}76 \cdot 9 \\
77 \cdot 2\end{array}$ & $\begin{array}{l}80 \cdot 1 \\
80 \cdot 2\end{array}$ & $\begin{array}{l}77 \cdot 6 \\
77 \cdot 8\end{array}$ & & & \\
\hline 6-11 hours & $\cdots$ & $\begin{array}{l}\mathbf{R} \\
\mathbf{L}\end{array}$ & $\begin{array}{l}78 \cdot 1 \\
78 \cdot 0\end{array}$ & $\begin{array}{l}78 \cdot 7 \\
78 \cdot 4\end{array}$ & $\begin{array}{l}79 \cdot 1 \\
79 \cdot 1\end{array}$ & $\begin{array}{l}79 \cdot 2 \\
78 \cdot 8\end{array}$ & & & $\begin{array}{l}78 \cdot 1-79 \cdot 2 \\
78 \cdot 0-79 \cdot 1\end{array}$ & $\begin{array}{l}78 \cdot 9 \\
78 \cdot 6\end{array}$ \\
\hline 12-23 hours & . & $\begin{array}{l}\mathbf{R} . \\
\text { L. }\end{array}$ & $\begin{array}{l}76 \cdot 1 \\
77 \cdot 3\end{array}$ & $\begin{array}{l}75 \cdot 6 \\
78 \cdot 3\end{array}$ & & & & & $\begin{array}{l}75 \cdot 6-76 \cdot 1 \\
77 \cdot 3-78 \cdot 3\end{array}$ & $\begin{array}{l}75 \cdot 8 \\
77 \cdot 8\end{array}$ \\
\hline 24-47 hours & $\cdots$ & $\begin{array}{l}\mathbf{R} . \\
\mathbf{L} .\end{array}$ & $\begin{array}{l}77 \cdot 6 \\
77 \cdot 7\end{array}$ & $\begin{array}{l}75 \cdot 2 \\
74 \cdot 9\end{array}$ & $\begin{array}{l}75 \cdot 2 \\
75 \cdot 9\end{array}$ & & & & $\begin{array}{l}75 \cdot 2-77 \cdot 6 \\
75 \cdot 9-77 \cdot 7\end{array}$ & $\begin{array}{l}76 \cdot 0 \\
76 \cdot 2\end{array}$ \\
\hline 48 hours to 3 day & & $\begin{array}{l}\mathbf{R} . \\
\mathbf{L} .\end{array}$ & $\begin{array}{l}74 \cdot 0 \\
75 \cdot 2\end{array}$ & $\begin{array}{l}75 \cdot 7 \\
76 \cdot 0\end{array}$ & $\begin{array}{l}76 \cdot 6 \\
77 \cdot 1\end{array}$ & $\begin{array}{l}75 \cdot 0 \\
75 \cdot 6\end{array}$ & $\begin{array}{l}77 \cdot 0 \\
78 \cdot 1\end{array}$ & & $\begin{array}{l}74 \cdot 0-77 \cdot 0 \\
75 \cdot 2-78 \cdot 1\end{array}$ & $\begin{array}{l}75 \cdot 7 \\
76 \cdot 4\end{array}$ \\
\hline [4-7 days & .. & $\begin{array}{l}\mathbf{R} . \\
\text { L. }\end{array}$ & $\begin{array}{l}74 \cdot 5 \\
76 \cdot 4\end{array}$ & $\begin{array}{l}78 \cdot 2 \\
77 \cdot 8\end{array}$ & $\begin{array}{l}77 \cdot 4 \\
77 \cdot 3\end{array}$ & & & & $\begin{array}{l}74 \cdot 5-78 \cdot 2 \\
76 \cdot 4-77 \cdot 8\end{array}$ & $\begin{array}{l}76 \cdot 7 \\
77 \cdot 8\end{array}$ \\
\hline [8-21 days &. & $\begin{array}{l}\mathbf{R} . \\
\text { L. }\end{array}$ & $\begin{array}{l}76 \cdot 6 \\
77 \cdot 6\end{array}$ & $\begin{array}{l}73 \cdot 0 \\
74 \cdot 4\end{array}$ & $\begin{array}{l}77 \cdot 8 \\
77 \cdot 8\end{array}$ & $\begin{array}{l}75 \cdot 3 \\
76 \cdot 4\end{array}$ & $75 \cdot 4$ & & $\begin{array}{l}73 \cdot 0-77 \cdot 8 \\
74 \cdot 4-77 \cdot 8\end{array}$ & $\begin{array}{l}75 \cdot 7 \\
76 \cdot 3\end{array}$ \\
\hline 21 days -5 weeks & $\cdots$ & $\begin{array}{l}\mathbf{R} . \\
\mathbf{L}\end{array}$ & $\begin{array}{l}77 \cdot 3 \\
77 \cdot 5\end{array}$ & $\begin{array}{l}76 \cdot 7 \\
77 \cdot 2\end{array}$ & $\begin{array}{l}75 \cdot 3 \\
75 \cdot 6\end{array}$ & $\begin{array}{l}72 \cdot 7 \\
72 \cdot 8\end{array}$ & $\begin{array}{l}73 \cdot 3 \\
72 \cdot 9\end{array}$ & $\begin{array}{l}76 \cdot 8 \\
76 \cdot 3\end{array}$ & $\begin{array}{l}72 \cdot 7-77 \cdot 3 \\
72 \cdot 8-77 \cdot 5\end{array}$ & $\begin{array}{l}76 \cdot 4 \\
76 \cdot 5\end{array}$ \\
\hline $6-8$ weeks & $\cdots$ & $\begin{array}{l}\mathbf{R} . \\
\mathbf{L} .\end{array}$ & $\begin{array}{l}78 \cdot 6 \\
78 \cdot 0\end{array}$ & $\begin{array}{l}75 \cdot 6 \\
75 \cdot 3\end{array}$ & $\begin{array}{l}76 \cdot 1 \\
75 \cdot 8\end{array}$ & $\begin{array}{l}75 \cdot 3 \\
76 \cdot 1\end{array}$ & $\begin{array}{l}75 \cdot 1 \\
75 \cdot 5\end{array}$ & $\begin{array}{l}74 \cdot 4 \\
74 \cdot 5\end{array}$ & $\begin{array}{l}74 \cdot 4-78 \cdot 6 \\
74 \cdot 5-78 \cdot 0\end{array}$ & $\begin{array}{l}75 \cdot 8 \\
75 \cdot 8\end{array}$ \\
\hline
\end{tabular}

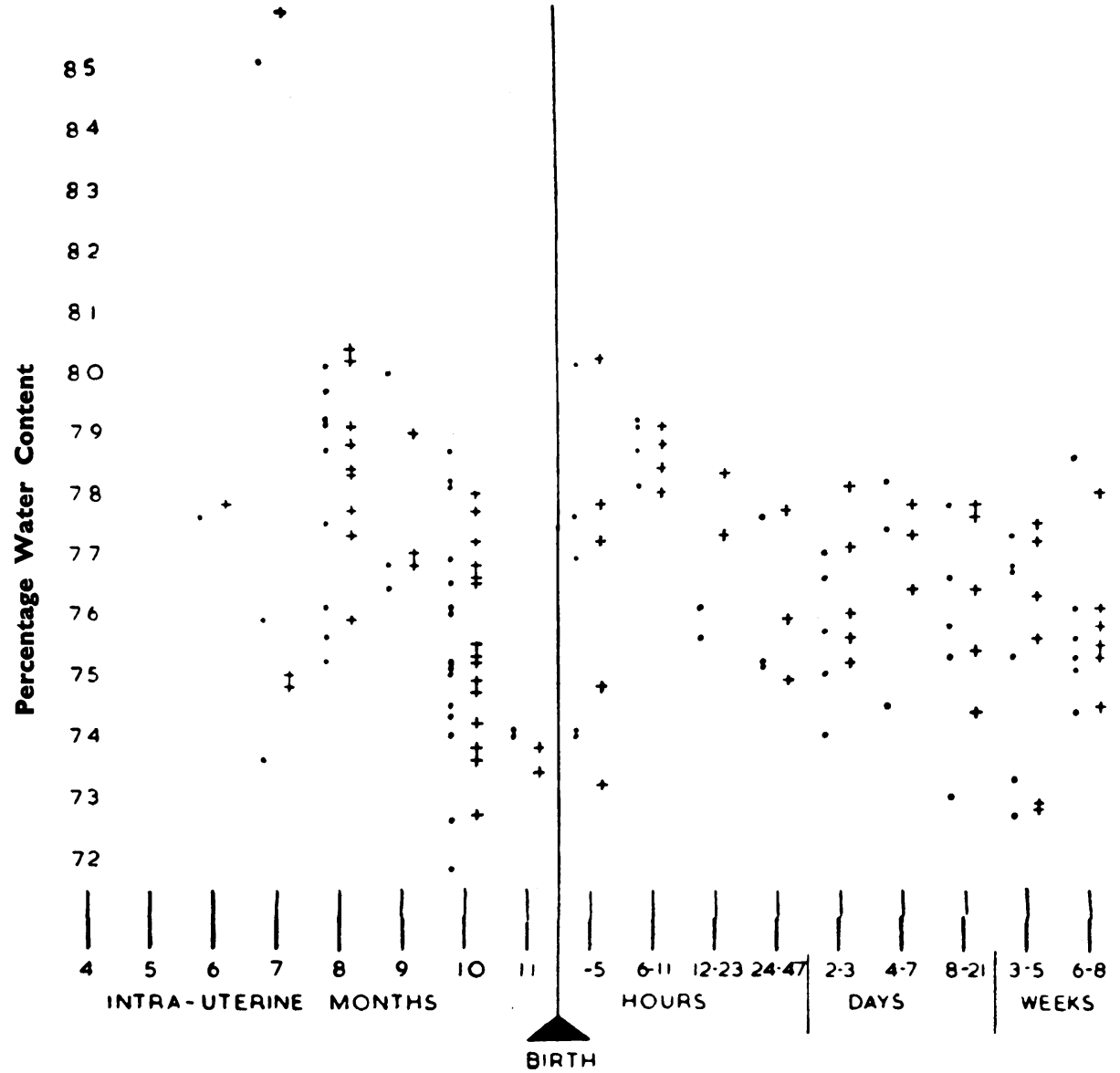

Spot $=$ Right Liver. $\quad+=$ Left Liver

Fig. 1.-Chart showing the distribution of percentage water content of 21 stillborns and 39 children dying after birth related to maturity and post-natal age. 
TABLE 3

FAT CONTENT BASED ON DRY WEIGHT OF THE RIGHT AND LEFT LIVERS OF INFANTS DYING BEFORE THE AGE OF 24 HOURS RELATED TO MATURITY

\begin{tabular}{|c|c|c|c|c|c|c|c|c|c|c|c|c|}
\hline $\begin{array}{l}\text { Maturity } \\
\text { (months) }\end{array}$ & \multicolumn{10}{|c|}{ Percentage Fat of Dried Weight } & Range & Average \\
\hline 4 & $\begin{array}{l}\mathbf{R} \text {. } \\
\mathbf{L} \text {. }\end{array}$ & $\begin{array}{l}8 \cdot 1 \\
8 \cdot 9\end{array}$ & $\begin{array}{r}7 \cdot 5 \\
13 \cdot 8\end{array}$ & $\begin{array}{l}9 \cdot 0 \\
9 \cdot 2\end{array}$ & & & & & & & $\begin{array}{l}7 \cdot 5-9 \cdot 0 \\
8 \cdot 9-13 \cdot 8\end{array}$ & $\begin{array}{r}8 \cdot 2 \\
10 \cdot 6\end{array}$ \\
\hline 5 & $\begin{array}{l}\mathbf{R} \text {. } \\
\mathbf{L} \text {. }\end{array}$ & $\begin{array}{l}7 \cdot 7 \\
7 \cdot 6\end{array}$ & & & & & & & & & - & $\begin{array}{l}7 \cdot 7 \\
7 \cdot 6\end{array}$ \\
\hline 6 & $\begin{array}{l}\mathbf{R} \text {. } \\
\mathbf{L} \text {. }\end{array}$ & $\begin{array}{r}10 \cdot 3 \\
9 \cdot 8\end{array}$ & & & & & & & & & - & $\begin{array}{r}10 \cdot 3 \\
9 \cdot 8\end{array}$ \\
\hline 7 & $\begin{array}{l}\mathbf{R} \text {. } \\
\mathbf{L} \text {. }\end{array}$ & $\begin{array}{l}11 \cdot 6 \\
12 \cdot 6\end{array}$ & $\begin{array}{l}18 \cdot 4 \\
19 \cdot 3\end{array}$ & $\begin{array}{l}8 \cdot 0 \\
9 \cdot 5\end{array}$ & & & & & & & $\begin{array}{l}8 \cdot 0-18 \cdot 4 \\
9 \cdot 5-19 \cdot 3\end{array}$ & $\begin{array}{l}12 \cdot 7 \\
10 \cdot 3\end{array}$ \\
\hline 8 & $\begin{array}{l}\mathbf{R} \text {. } \\
\mathbf{L} \text {. }\end{array}$ & $\begin{array}{l}7 \cdot 0 \\
9 \cdot 6\end{array}$ & $\begin{array}{l}10 \cdot 7 \\
11 \cdot 4\end{array}$ & $\begin{array}{l}5 \cdot 7 \\
5 \cdot 8\end{array}$ & $\begin{array}{l}7 \cdot 3 \\
7 \cdot 5\end{array}$ & $\begin{array}{l}8 \cdot 2 \\
6 \cdot 8\end{array}$ & $\begin{array}{l}10 \cdot 0 \\
10 \cdot 0\end{array}$ & $\begin{array}{l}11 \cdot 5 \\
11 \cdot 1\end{array}$ & $\begin{array}{l}12 \cdot 2 \\
11 \cdot 9\end{array}$ & $\begin{array}{l}12 \cdot 3 \\
13 \cdot 1\end{array}$ & $\begin{array}{l}7 \cdot 0-12 \cdot 2 \\
5 \cdot 8-11 \cdot 9\end{array}$ & $\begin{array}{l}9 \cdot 4 \\
9 \cdot 7\end{array}$ \\
\hline 9 & $\begin{array}{l}\mathbf{R} \text {. } \\
\text { L. }\end{array}$ & $\begin{array}{l}6 \cdot 2 \\
5 \cdot 4\end{array}$ & $\begin{array}{l}12 \cdot 9 \\
14 \cdot 9\end{array}$ & $\begin{array}{l}10 \cdot 9 \\
11 \cdot 7\end{array}$ & & & & & & & $\begin{array}{l}6 \cdot 2-12 \cdot 9 \\
5 \cdot 4-14 \cdot 9\end{array}$ & $\begin{array}{l}10 \cdot 0 \\
10 \cdot 3\end{array}$ \\
\hline 10 & $\begin{array}{l}\mathbf{R} . \\
\mathbf{L} .\end{array}$ & $\begin{array}{l}14 \cdot 2 \\
14 \cdot 0\end{array}$ & $\begin{array}{l}15 \cdot 3 \\
12 \cdot 1\end{array}$ & $\begin{array}{l}11 \cdot 5 \\
12 \cdot 6\end{array}$ & $\begin{array}{l}8 \cdot 1 \\
8 \cdot 7\end{array}$ & $\begin{array}{r}11 \cdot 1 \\
8 \cdot 4\end{array}$ & $\begin{array}{l}11 \cdot 7 \\
10 \cdot 5\end{array}$ & $\begin{array}{r}10.0 \\
9.0\end{array}$ & $\begin{array}{l}7 \cdot 8 \\
7 \cdot 8\end{array}$ & $\begin{array}{l}10 \cdot 7 \\
10 \cdot 5\end{array}$ & $\begin{array}{l}7 \cdot 6-15 \cdot 3 \\
6 \cdot 1-14 \cdot 0\end{array}$ & $\begin{array}{r}10 \cdot 8 \\
9 \cdot 9\end{array}$ \\
\hline & $\begin{array}{l}\mathbf{R} \text {. } \\
\mathbf{L} \text {. }\end{array}$ & $\begin{array}{l}12 \cdot 6 \\
10 \cdot 3\end{array}$ & $\begin{array}{l}11 \cdot 7 \\
10 \cdot 0\end{array}$ & $\begin{array}{l}9 \cdot 3 \\
9 \cdot 4\end{array}$ & $\begin{array}{r}9 \cdot 2 \\
10 \cdot 0\end{array}$ & $\begin{array}{l}7 \cdot 6 \\
6 \cdot 1\end{array}$ & $\begin{array}{r}11 \cdot 6 \\
9 \cdot 3\end{array}$ & & & & $9.5-11.5$ & \\
\hline 11 & $\begin{array}{l}\mathbf{R} . \\
\mathbf{L} .\end{array}$ & $\begin{array}{r}9 \cdot 5 \\
10 \cdot 0\end{array}$ & $\begin{array}{l}11 \cdot 5 \\
10 \cdot 0\end{array}$ & & & & & & & & - & $\begin{array}{l}10 \cdot 5 \\
10 \cdot 0\end{array}$ \\
\hline
\end{tabular}

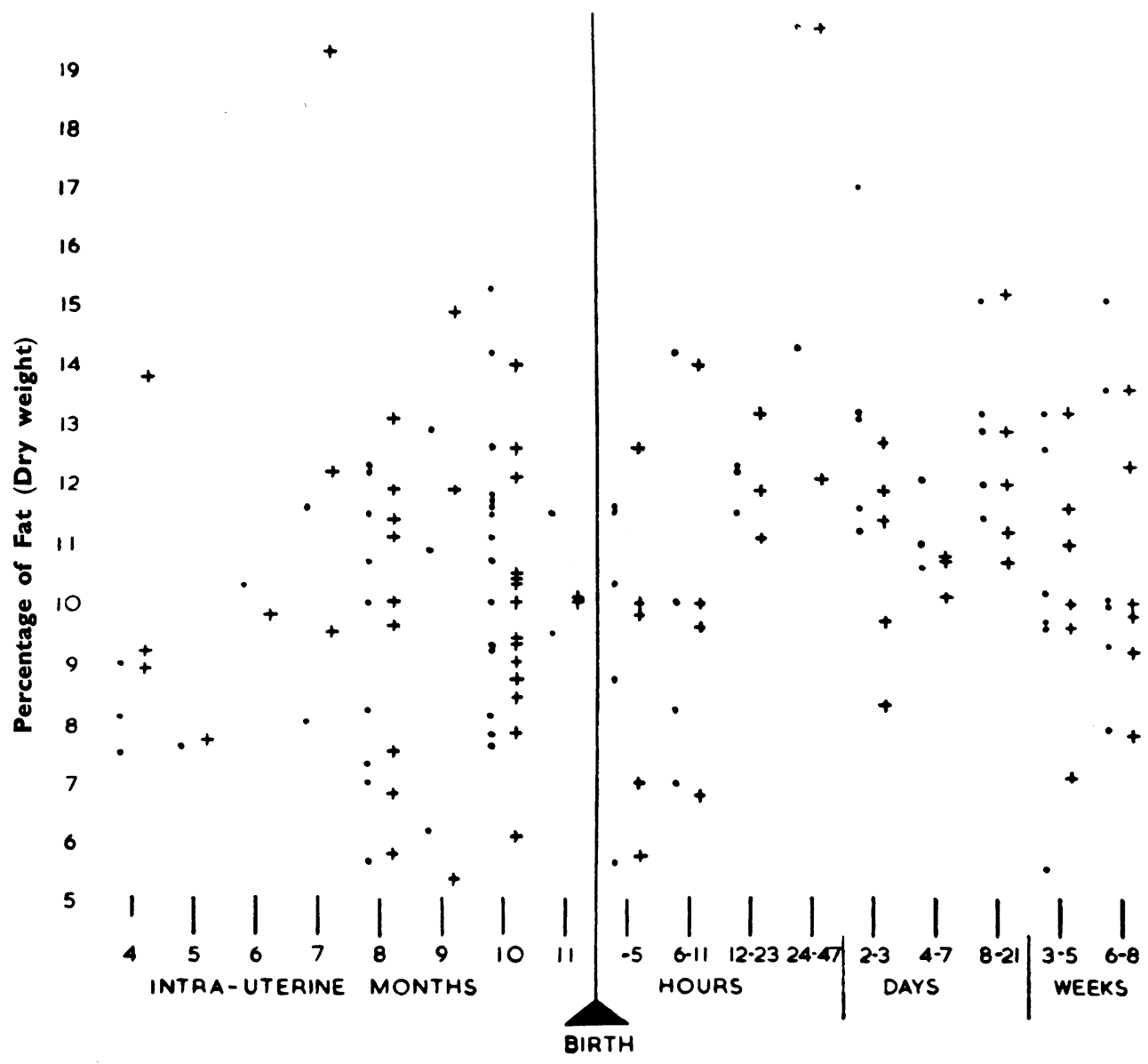

Spot $=$ Right Liver. $++=$ Left Liver.
FIG. 2.-Spot graph showing the distribution of percentage (dried weight) at content of 25 stillborn and 39 children dying after birth, related to intra-uterine maturity and post-natal age. 
TABLE 4

FAT CONTENT BASED ON DRY WEIGHT OF THE RIGHT AND LEFT LOBES IN HISTOLOGICALLY NORMAL LIVERS OF CHILDREN DYING AT DIFFERENT AGES AFTER BIRTH

\begin{tabular}{|c|c|c|c|c|c|c|c|c|c|c|}
\hline \multicolumn{2}{|l|}{ Age } & \multicolumn{7}{|c|}{ Percentage Fat Content } & \multirow{2}{*}{$\begin{array}{l}\text { Range } \\
5 \cdot 7-11 \cdot 5 \\
5 \cdot 8-12 \cdot 6\end{array}$} & \multirow{2}{*}{$\begin{array}{c}\text { Average } \\
\begin{array}{c}9 \cdot 3 \\
9 \cdot 0\end{array}\end{array}$} \\
\hline Birth to 5 hours & . & $\begin{array}{l}\text { R. } \\
\text { L. }\end{array}$ & $\begin{array}{l}5 \cdot 7 \\
5 \cdot 8\end{array}$ & $\begin{array}{l}11 \cdot 5 \\
12 \cdot 6\end{array}$ & $\begin{array}{l}11 \cdot 5 \\
10.0\end{array}$ & $\begin{array}{l}7 \cdot 7 \\
7 \cdot 0\end{array}$ & $\begin{array}{r}10 \cdot 3 \\
9 \cdot 8\end{array}$ & & & \\
\hline $6-11$ hours & . & $\begin{array}{l}\mathbf{R} . \\
\mathbf{L} .\end{array}$ & $\begin{array}{l}14 \cdot 2 \\
14 \cdot 0\end{array}$ & $\begin{array}{l}7 \cdot 0 \\
9 \cdot 6\end{array}$ & $\begin{array}{l}10 \cdot 0 \\
10 \cdot 0\end{array}$ & $\begin{array}{l}8 \cdot 2 \\
6 \cdot 8\end{array}$ & & & $\begin{array}{l}7 \cdot 0-14 \cdot 2 \\
6 \cdot 8-14 \cdot 0\end{array}$ & $\begin{array}{r}9 \cdot 9 \\
10 \cdot 1\end{array}$ \\
\hline 12-23 hours & $\cdots$ & $\begin{array}{l}\mathbf{R} . \\
\mathbf{L} .\end{array}$ & $\begin{array}{l}12 \cdot 3 \\
13 \cdot 1\end{array}$ & $\begin{array}{l}12 \cdot 2 \\
11 \cdot 9\end{array}$ & $\begin{array}{l}11 \cdot 5 \\
11 \cdot 1\end{array}$ & & & & $\begin{array}{l}11 \cdot 5-12 \cdot 3 \\
11 \cdot 1-13 \cdot 1\end{array}$ & $\begin{array}{l}12 \cdot 0 \\
12 \cdot 0\end{array}$ \\
\hline 24-47 hours & $\cdots$ & $\begin{array}{l}\mathbf{R} . \\
\mathbf{L} .\end{array}$ & $\begin{array}{l}15 \cdot 3 \\
12 \cdot 1\end{array}$ & $\begin{array}{l}19 \cdot 7 \\
19 \cdot 7\end{array}$ & & & & & $\begin{array}{l}15 \cdot 3-19 \cdot 7 \\
12 \cdot 1-19 \cdot 7\end{array}$ & $\begin{array}{l}17 \cdot 5 \\
15 \cdot 9\end{array}$ \\
\hline 48 hours to 3 day & & $\begin{array}{l}\mathbf{R} . \\
\mathbf{L} .\end{array}$ & $\begin{array}{l}17 \cdot 0 \\
11 \cdot 9\end{array}$ & $\begin{array}{r}13 \cdot 2 \\
8 \cdot 3\end{array}$ & $\begin{array}{l}13 \cdot 1 \\
12 \cdot 7\end{array}$ & $\begin{array}{r}11 \cdot 6 \\
9 \cdot 7\end{array}$ & $\begin{array}{l}11 \cdot 2 \\
11 \cdot 4\end{array}$ & & $\begin{array}{r}11 \cdot 2-17 \cdot 0 \\
8 \cdot 3-12 \cdot 7\end{array}$ & $\begin{array}{l}13 \cdot 2 \\
10 \cdot 8\end{array}$ \\
\hline 4-7 days & $\cdots$ & $\begin{array}{l}\mathbf{R} . \\
\mathbf{L} .\end{array}$ & $\begin{array}{l}10 \cdot 6 \\
10 \cdot 1\end{array}$ & $\begin{array}{l}11 \cdot 0 \\
10 \cdot 7\end{array}$ & $\begin{array}{l}12 \cdot 1 \\
10 \cdot 8\end{array}$ & & & & $\begin{array}{l}10 \cdot 6-12 \cdot 1 \\
10 \cdot 1-10 \cdot 8\end{array}$ & $\begin{array}{l}11 \cdot 2 \\
10 \cdot 5\end{array}$ \\
\hline 8-20 days & . & $\begin{array}{l}\text { R. } \\
\mathbf{L} .\end{array}$ & $\begin{array}{l}12 \cdot 0 \\
11 \cdot 2\end{array}$ & $\begin{array}{l}15 \cdot 1 \\
15 \cdot 2\end{array}$ & $\begin{array}{l}12 \cdot 9 \\
12 \cdot 9\end{array}$ & $\begin{array}{l}11 \cdot 4 \\
10 \cdot 7\end{array}$ & $\begin{array}{l}13 \cdot 2 \\
12 \cdot 0\end{array}$ & & $\begin{array}{l}11 \cdot 4-15 \cdot 1 \\
10 \cdot 7-15 \cdot 2\end{array}$ & $\begin{array}{l}16 \cdot 1 \\
15 \cdot 5\end{array}$ \\
\hline 31 days to 5 weel & & $\begin{array}{l}\text { R. } \\
\text { L. }\end{array}$ & $\begin{array}{r}9 \cdot 7 \\
10 \cdot 0\end{array}$ & $\begin{array}{l}5 \cdot 7 \\
7 \cdot 1\end{array}$ & $\begin{array}{l}13 \cdot 2 \\
13 \cdot 2\end{array}$ & $\begin{array}{l}10 \cdot 2 \\
11 \cdot 0\end{array}$ & $\begin{array}{l}12 \cdot 6 \\
11 \cdot 6\end{array}$ & $\begin{array}{l}9 \cdot 6 \\
9 \cdot 6\end{array}$ & $\begin{array}{l}5 \cdot 7-13 \cdot 2 \\
7 \cdot 1-13 \cdot 2\end{array}$ & $\begin{array}{l}10 \cdot 2 \\
10 \cdot 4\end{array}$ \\
\hline 6-8 weeks & . & $\begin{array}{l}\mathbf{R} \text {. } \\
\mathbf{L} \text {. }\end{array}$ & $\begin{array}{l}14 \cdot 1 \\
12 \cdot 6\end{array}$ & $\begin{array}{l}10 \cdot 0 \\
10 \cdot 0\end{array}$ & $\begin{array}{l}13 \cdot 6 \\
12 \cdot 3\end{array}$ & $\begin{array}{l}9 \cdot 3 \\
9 \cdot 2\end{array}$ & $\begin{array}{r}10 \cdot 0 \\
9 \cdot 8\end{array}$ & $\begin{array}{l}7 \cdot 9 \\
7 \cdot 8\end{array}$ & $\begin{array}{l}9 \cdot 3-14 \cdot 1 \\
9 \cdot 2-12 \cdot 6\end{array}$ & $\begin{array}{l}10 \cdot 8 \\
10 \cdot 3\end{array}$ \\
\hline
\end{tabular}

Fat Content in Livers in Utero. The fat content of these apparently normal livers showed a greater variation than the water content. The complete range found was from $5 \cdot 4$ to $19 \cdot 3 \%$. Study of the figures (Table 3) and of the distribution of levels (Fig. 2) suggested that variation in fat content during foetal life was considerably less than the variation found at any of the later stages of development. The individual difference between the fat contents of the different lobes was up to $2 \cdot 7 \mathrm{~g}$. $\%$-a difference very small when compared with the difference frequently found in abnormal livers.

Fat Content of Livers after Birth. The fat content of the liver after birth showed a wide range similar to that seen in stillborn infants (Table 4).

While the individual difference in fat content between the left and right livers was not great, there appeared to be a tendency for the right liver to have a slightly higher fat content than the left in infants surviving more than 12 hours.

The livers of lowest fat content, 5-7 g. $\%$, were found in infants dying within six hours of birth.

\section{Discussion}

The purpose of this study was to establish some data of the normal water and fat content of the right and left lobes of the liver in the foetus and newborn. Since all of the material studied came from infants who had died, the criteria of normality are arbitrary and based on personal naked eye and microscopic opinion of the livers and of the cause of death of the children concerned.
The criteria used tended to reveal abnormalities of over-hydration and increase in fat content rather than those of the reverse aspect. Fatty vacuolation is easily seen, but there is no similar simple criterion to exclude livers containing abnormally little fat, so we feel that the lower ranges of fat content found were probably from infants with unrecognized general diseases. Also minor differences in the size and staining properties of cells tend to be more obvious when seen adjacently and in the same microscopic preparation. This suggests that selection of cases may have excluded livers showing minor local asymmetrical disease and at the same time admit livers having perhaps a greater generalized deficiency abnormality. For these reasons the ranges of normality can probably be assessed better by general inspection of the figures than by statistical study.

The figures for fat content showed wide variation. If for the reasons enumerated above we ignore the lowest fat levels, i.e., below $7 \%$, and the single liver with fat levels above $18 \%$, there seems to be very little variation in fat content through the whole period studied but the range is over $100 \%$, i.e., from 7 to $15 \mathrm{~g}$. \%. This is not surprising as Leathes (1909) noted great variability in the content of the livers of slaughtered animals quite independent of the presence of fatty vacuolation.

Dorkin and Weinberg (1949) found much histologically demonstrable fat in the livers of infants over the weight of $2,425 \mathrm{~g}$. and in infants below $2,000 \mathrm{~g}$. either no fat or an occasional droplet. They concluded that fatty vacuolation in the liver is 
normal in the newborn. We do not accept their assumption and this study is concerned only with livers showing no fatty vacuolation. Imrie and Graham (1920) showed that in guinea-pigs the fat content of the liver rose during the latter part of intra-uterine life and fell to normal adult levels within 80 hours of birth. Imrie (1914) had examined the fat contents of the liver in two foetuses showing a fat content of $1.38 \%$ (wet weight) at six months' and $2.58 \%$ at seven months' gestation. The fat content of two infants dying at 2 days were 1.90 and $3 \cdot 18 \%$.

Regarding the difference between the left and right lobes of the liver, the technical errors of the method used suggested that a difference of over $1.88 \mathrm{~g}$. $\%$ might be significant. The ranges found in each lobe in each age group are very wide, and, as the mean levels in the groups containing the largest number of specimens show differences of no significance, it would seem that there is normally no difference between the fat content of the left and right livers at different stages of maturity. After birth there is no apparent difference in the fat content of the lobes during the first 24 hours, but in infants dying during the rest of the first week there appears to be a tendency for the fat content of the right liver to be higher than the left, the difference between the lobes being up to $5 \cdot 7 \mathrm{~g}$. $\%$.

Our results indicate at no time a significant difference in water content between the left and right lobes of the liver. In the foetus of nine months' gestation or less the water content ranged from 75 to $80 \%$. In the mature infant it would seem to be approximately $2 \%$ less. If the 12 hours following birth are ignored the water content of the liver apparently remains fairly constant between $74 \%$ and $78 \%$ throughout the neonatal period.

\section{Summary}

The fat and water content of the left and right physiological lobes of apparently normal livers has been estimated in stillborn babies and infants dying in the first few weeks after birth.

No difference in water content was found between the left and right lobes either in stillborn babies of different ages after birth. The range of water content up to eight months' gestation is apparently between 75 and $80 \%$. In the mature child and after birth the range is approximately $2 \%$ lower.

The fat content of the livers showed a much greater variation than water content, the normal range of fat content being 7-15 g. \% dry weight. There appeared to be no significant difference between the fat content of the left and right livers when related to maturity but a tendency for the fat content of the right livers to be higher than the left in infants dying between the ages of 1 hour and 7 days.

REFERENCES

Billing, B. H., Conlon, H. J., Hein, D. E., and Schiff, L. (1953). J. clin. Invest., 32, 214.

Dorkin, J. R. and Weinberg, T. (1949). Arch. Path., Chicago, 48, 578.

Emery, J. L. (1952) Archives of Disease in Childhood, 27, 558.

Gruenwald, P. (1949). Amer. J. clin. Path., 19, 801.

Imrie, C. G. (1914). J. Path. Bact., 19, 245.

and Graham, S. G. (1920), J. biol. Chem., 44, 243.

Leathes, J. B. (1909). Lancet, 1, 593.

Needham, J. (1931). Chemical Embryology, Vol. 2, p. 914, Fig. 245. Cambridge.

Rourke, G. M. and Stewart, J. D. (1942). Arch. Path., Chicago, 33,603 .

Scammon, R. E. and Calkins, L. A. (1923). Proc. Soc. exp. Biol., N.Y., 20, 353 .

Streeter, G. L. (1920). Contr. Embryol. Carneg. Instn., 11, (No. 55), 143.

Widdowson, E. M. and Spray, C. M. (1951). Archives of Disease in Childhood, 26, 205. 